\title{
Lymphangioma circumscriptum als Folge einer chirurgisch-radiologischen Therapie genitaler Malignome
}

G. Wagner

\author{
Lymphangioma Circumscriptum as a Result of Combined Surgical-Radiological \\ Therapy of Genital Malignoma
}

\section{Zusammenfassung}

Am Beispiel der Kasuistiken von drei Patienten wird das Lymphangioma circumscriptum als Folge onkologischer Therapieverfahren beschrieben. Alle Patienten hatten sich einer chirurgischradiologischen Behandlung genitaler Malignome unterziehen müssen. Die Latenzzeit zwischen der onkologischen Therapie und dem Auftreten der Dermatose am Penis, am Skrotum oder an der Vulva betrug 2 - 29 Jahre. Neben der Manifestation bei genitalen Malignomen wurde das Lymphangioma circumscriptum typischerweise auch nach der Behandlung von Mammakarzinomen beobachtet. Die Therapie anderer Tumorformen oder entzündliche Grunderkrankungen müssen hingegen als sehr seltene Ursachen eines erworbenen Lymphangioma circumscriptum angesehen werden.

\section{Abstract}

Three patients with lymphangioma circumscriptum are described who suffered from this disease as a result of oncological therapy. All patients were treated by surgical-radiological procedures. The latent period between oncological treatment and first signs of lymphangioma circumscriptum of the penis, the scrotum or the vulva was 2-29 years. Lymphangioma circumscriptum has been observed in combination with genital malignoma as well as after treatment of breast cancer. In rare cases, however, it has been described in combination with other tumors and chronic inflammatory diseases.

\section{Einleitung}

Das Lymphangioma circumscriptum kann bei weitgehend identischer klinischer Morphologie in zwei unterschiedlichen Verlaufsformen beobachtet werden. Die kongenitale oder im frühen Kindesalter auftretende Manifestation, die auch als primäre Form bezeichnet wird, zählt zu den Hamartomen und ist nicht selten mit weiteren Fehlbildungen des Lymphgefäßsystems verbunden. Hierzu zählen die Lymphangiomatose, kavernöse Lymphangiome oder zystische Hygrome [1]. Demgegenüber findet sich das Lymphangioma circumscriptum im Erwachsenenalter, die erworbene oder sekundäre Manifestationsform, in der Regel auf dem Boden eines chronischen Lymphödems als Folge einer vorausgegangenen onkologischen Therapie. Während die Frage nach der Pathogenese der Lymphangiome bei der primären Form weiterhin umstritten ist, diskutiert werden eine neoplastische oder eine ektatische Genese, besteht bei der erworbenen Manifestationsform die einheitliche Auffassung, dass hier reaktive Lymphangiektasien vorliegen [1-3]. Bei den nachfolgenden drei Kasuistiken konnte die Schädigung des Lymphgefäßsystems auf eine kombinierte chirurgisch-radiologische Behandlung genitaler Malignome zurückgeführt werden, wie dies auch für fast alle anderen in der Literatur beschriebenen Erkrankungsfälle erworbener zirkumskripter Lymphangiome typisch gewesen ist. 


\section{Patient K. S.}

Im Alter von 38 Jahren wurde bei dem jetzt 70-jährigen Patienten ein Peniskarzinom diagnostiziert. Neben der Amputation des Penis erfolgte eine inguinale Lymphadenektomie bds. und eine Radiatio des gesamten Operationsgebietes. 29 Jahre nach dieser Behandlung bemerkte er erstmals nässende Hautveränderungen am Skrotum. Klinisch fand sich ein vergrößertes, derbes Skrotum mit deutlich abgeflachten Krypten. Im Bereich des gesamten Skrotums zeigten sich einzelne oder gruppiert stehende, hier auch konfluierende, 2-6 mm durchmessende, teilweise breitbasig gestielte Papeln mit jeweils mehreren unterschiedlich großen Bläschen. Die überwiegend klare Flüssigkeit der Bläschen war vereinzelt pustulös eingetrübt (Abb.1,2).

\section{Patient H. A.}

Bei dem heute 76-jährigen Patienten wurde vor 40 Jahren eine Orchiektomie durchgeführt, nachdem sich ein Seminom am rechten Hoden entwickelt hatte. Anschließend erfolgte eine Kobalt-Bestrahlung des Skrotums und der abfließenden Lymphbahnen. Bereits zwei Jahre nach dieser Behandlung traten Hautveränderungen am Penis und am Skrotum auf. Der dermatologische Befund zeichnete sich durch überwiegend gruppiert stehende, vereinzelt konfluierende, 2 - $3 \mathrm{~mm}$ große, derbe Bläschen aus. Insbesondere die Haut des Penis war teigig-ödematös geschwollen mit tiefen, ringförmigen Falten (Abb. 3).

\section{Patientin W. M.}

Die 85-jährige Patientin erkrankte 1978 an einem Cervixkarzinom. Therapeutisch erfolgte eine Hysterektomie mit nachfolgender Radium-Einlage. Ein Jahr später wurde ein Plattenepithelkarzinom der rechten Vulva exzidiert mit anschließender Radiatio des Op.-Bereiches. Im Frühjahr 2004 waren ihrem Gynäkologen erstmals Hautveränderungen aufgefallen. Bei der dermatologischen Untersuchung war die Vulvaschleimhaut bei Zustand nach Vulvektomie rechts nur noch linksseitig in stark atrophischer Form erkennbar. Die angrenzenden Hautbereiche waren bds. ödematös und zeigten hier gruppiert stehende, in der Mehrzahl nur sehr kleine und flache, $2-3 \mathrm{~mm}$ durchmessende Bläschen (Abb. 4).

Bei keinem der drei Patienten war es nach der onkologischen Behandlung zu Rezidiven oder Metastasierungen der jeweiligen Malignome gekommen. Auch das Auftreten von Erysipelen oder anderen urogenitalen Infektionen wurde von den Patienten verneint.

Die histopathologischen Befunde der drei Patienten waren weitgehend identisch. Dabei zeigte sich die zentral vorgewölbte Epidermis entweder akanthotisch verbreitert mit Hypergranulose und kompakter Orthokeratose oder war deutlich verschmälert. Subepidermal fanden sich wabenartige, stark dilatierte Hohlräume mit einem dünnen Endothel und fixierter Lymphe. Im angrenzenden fibrosierten Stroma konnten teilweise ausgeprägte Lymphangiektasien nachgewiesen werden (Dr. C. Diaz, Einsendungslabor für Dermatopathologie, Freiburg; Abb. 5).

Eine Behandlung wurde von dem Patienten K. S. und der Patientin W. M. nicht gewünscht. Bei dem Pat. H. A. wurde ein um-

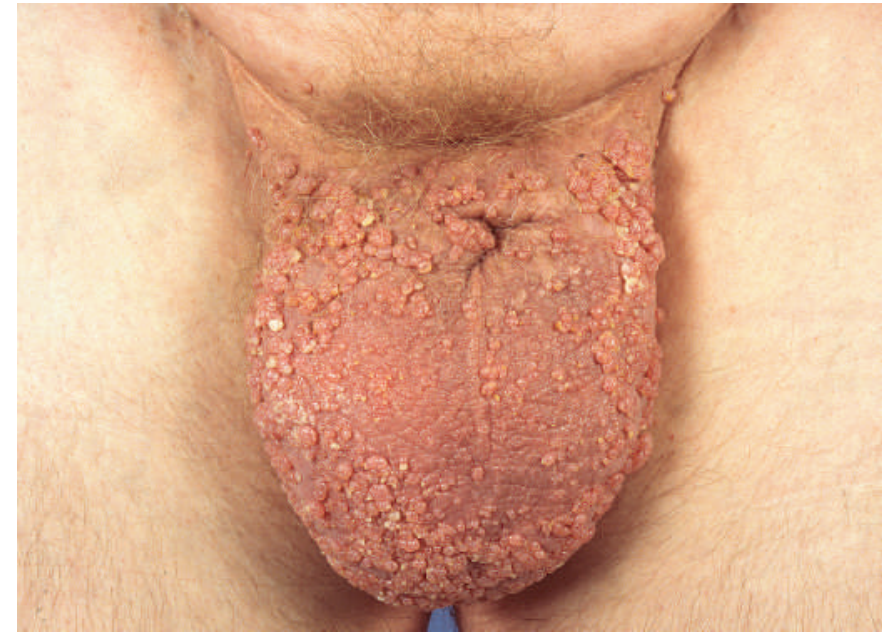

Abb. 1 Patient K. S.: Dicht stehende Papulovesikel, Z. n. Penisamputation.

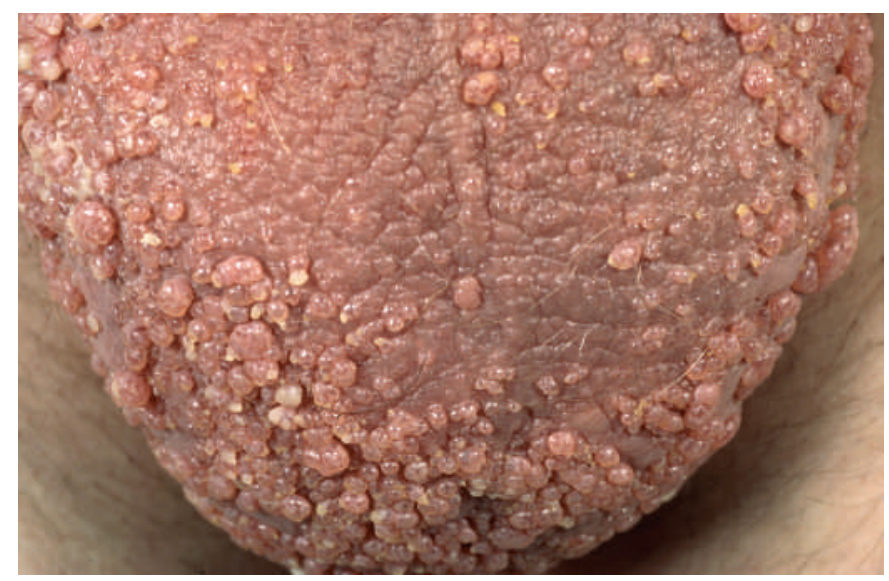

Abb. 2 Patient K. S.: Detailaufnahme.

schriebenes Areal mit dem Argon-Laser behandelt, was zu einer anhaltenden Rückbildung der Bläschen führte. Eine weitere Laser-Therapie lehnte der Patient dann jedoch ab.

\section{Diskussion}

Das Lymphangioma circumscriptum ist auch in seiner erworbenen Form eine nur selten beobachtete Dermatose, über die in der Literatur bisher hauptsächlich in kasuistischen Beiträgen berichtet worden ist. Epidemiologische Daten fehlen daher ebenso wie konkrete Angaben zu den Behandlungsverfahren der meist vorbestehenden Malignome, z.B. zu den eingesetzten Strahlendosierungen, die nur selten dokumentiert worden sind [4]. Vereinzelt wurden zu speziellen Fragestellungen Literaturuntersuchungen durchgeführt. Als Beispiel sei auf eine Arbeit von Vlastos et al. hingewiesen [5]. Die Autoren führten anhand der Literatur eine Datenanalyse durch, wie häufig über das Lymphangioma circumscriptum im Bereich der Vulva berichtet worden ist. Dabei fanden sich in einer Gruppe von 3272 Arbeiten über Lymphangiome, die in den Jahren von 1961 bis 2003 publiziert worden waren, 11 Arbeiten über primäre und 20 Publikationen über sekundäre Lymphangiomata circumscripta im Bereich der Vulva. 


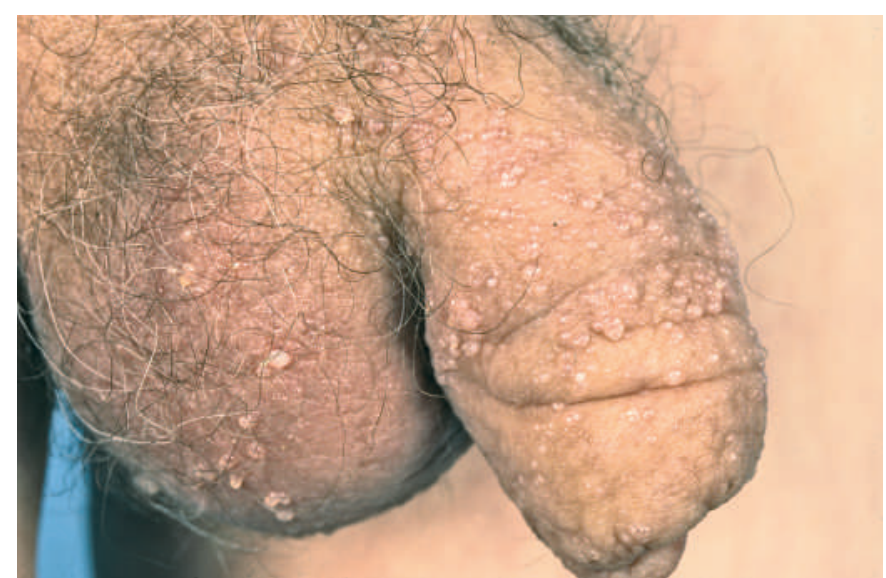

Abb. 3 Patient H. A.: Einzeln stehende oder konfluierende Vesikel Penis und Skrotum.

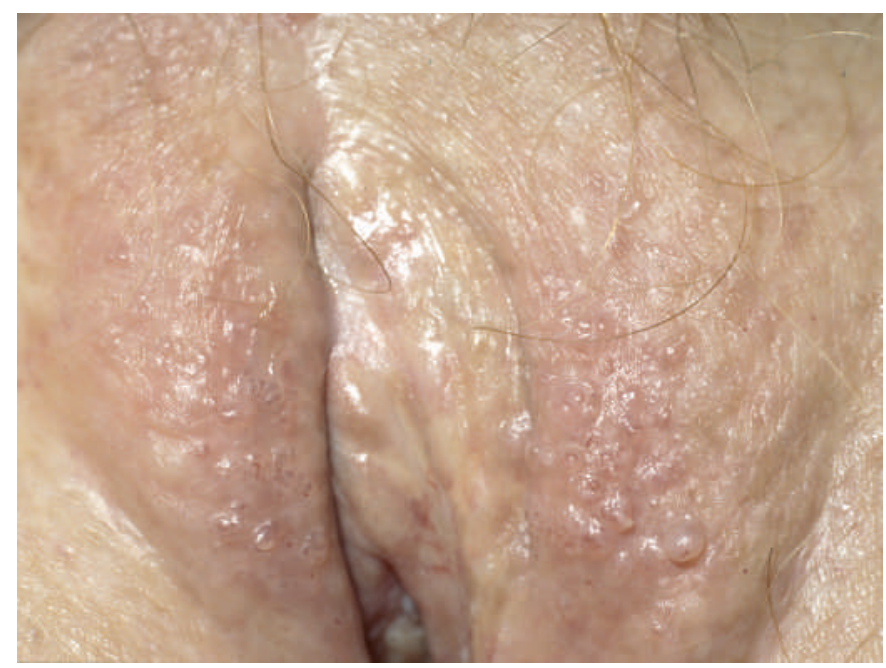

Abb. 4 Patientin W. M.: Gruppiert stehende kleinste Vesikel im Vulvabereich.

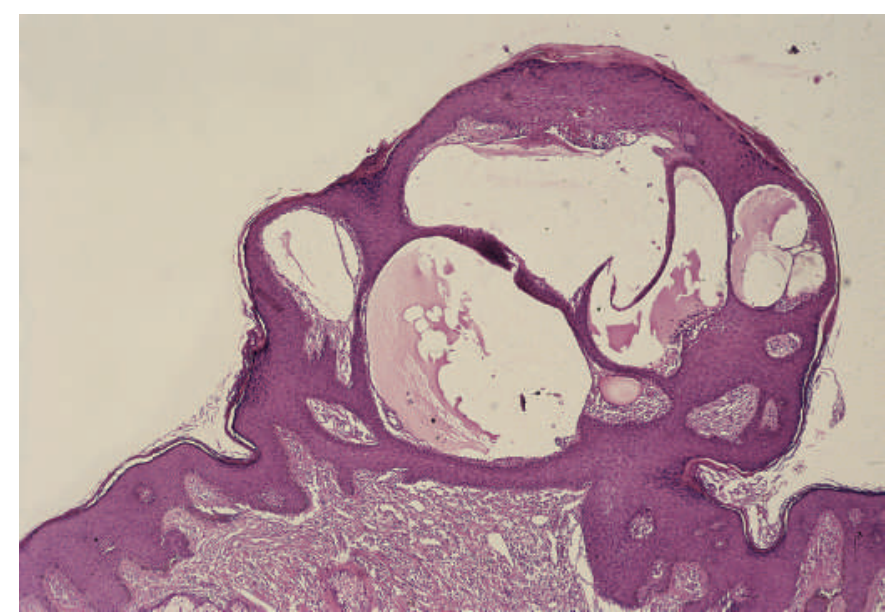

Abb. 5 Subepidermale Lymphangiektasien (HE, 1:40).
Der spezielle Aspekt einer solchen Untersuchung erlaubt somit jedoch keine allgemein gültigen Aussagen zu epidemiologischen Daten des Lymphangioma circumscriptum.

Während sich das primäre Lymphangioma circumscriptum typischerweise am oberen Stamm manifestiert, zeigen sich die erworbenen Formen in Abhängigkeit von der behandelten Tumorform genital, inguinal und pektoral, wobei dann üblicherweise das Bestrahlungsareal oder der unmittelbare Bereich distal der ursprünglichen Op.-Lokalisation betroffen sind [5]. Die Latenzzeit zwischen der onkologischen Therapie und dem Auftreten der zirkumskripten Angiome wird in den meisten Fällen mit wenigen Jahren, vereinzelt aber auch mit mehreren Jahrzehnten angegeben $[3,6-9]$.

Die klinische Morphologie der erworbenen Form des Lymphangioma circumscriptum ist durch gruppiert stehende, linsen- bis halbkirschkerngroße Bläschen gekennzeichnet, die eine wasserklare oder hellgelbe Flüssigkeit enthalten $[4,8,10]$. Hämorrhagische Bläschen als Ausdruck einer sekundären Blutung, wie sie bei der primären Form des Lymphangioma circumscriptum häufig vorliegt, wurden bei der erworbenen Manifestationsform nur selten beschrieben $[1,6]$. So fanden sich auch bei unseren Patienten keine hämorrhagischen Bläschen. Dafür zeigten sich vereinzelt pustulöse Eintrübungen, die als Zeichen einer bakteriellen Infektion gewertet wurden. Eine weitere morphologische Eigenschaft, die besonders das klinische Bild der erworbenen Form des Lymphangioma circumscriptum prägt, ist die Neigung der einzelnen Bläschen, zu größeren, dann auch mehrkammerigen Blasen zu konfluieren [6]. Der Palpationsbefund der Blasen ist dabei typischerweise prall-elastisch. Bei dünnen Blasendecken und verstärkter Vulnerabilität kann die austretende Lymphflüssigkeit kumulativ-toxische Kontaktekzeme verursachen oder bakterielle Infektionen begünstigen. Vereinzelt, wie z.B. bei dem hier vorgestellten Patienten K. S., zeigen die Morphen eine ausgeprägt papulöse Komponente, wobei die meist kleinen Blasen dennoch klinisch sichtbar bleiben [11]. Fehlen blasige Anteile hingegen vollständig, kann der dermatologische Befund auch durch hyperpigmentierte oder verruköse Papeln gekennzeichnet sein [6-8]. Die beschriebenen Morphen der erworbenen Form des Lymphangioma circumscriptum finden sich häufig auf dem Boden chronischer Lymphödeme. Da Lymphödeme jedoch keineswegs bei allen Patienten beobachtet werden, scheinen sie keine unabdingbare Voraussetzung für die Entstehung eines Lymphangioma circumscriptum darzustellen [3,6,8 - 10]. Das erworbene Lymphangioma circumscriptum ist definitionsgemäß die Folge einer vorbestehenden Erkrankung oder der damit notwendig gewordenen Therapie. In den meisten Fällen hatten sich die Patienten einer kombinierten chirurgisch-radiologischen Behandlung einer Tumorerkrankung unterziehen müssen, so auch die hier vorgestellten Patienten. In Ausnahmefällen wurde das Lymphangioma circumscriptum allerdings auch beobachtet, ohne dass eine Kombination der genannten Therapieverfahren erfolgt war $[4,12]$. Diese Patienten waren dann entweder operiert oder bestrahlt worden. Die typischen Malignome, infolge deren Therapie das Lymphangioma circumscriptum auftreten kann, sind genitale Karzinome, besonders das Cervixkarzinom, sowie das Mammakarzinom [6 - 10]. Die Behandlung anderer Tumorformen oder entzündliche Grunderkrankungen müssen hingegen als sehr seltene Ursachen eines Lymphangioma circums- 
criptum angesehen werden. $\mathrm{Zu}$ diesen Einzelbeobachtungen zählen z.B. ein malignes Melanom der unteren Extremitäten, bei dem eine inguinale Lymphadenektomie und eine hypertherme Zytostatika-Perfusion durchgeführt worden waren, oder eine radiologische Therapie als Prophylaxe von Keloiden [3]. Gelegentlich ist das Lymphangioma circumscriptum auch bei internistischen Grunderkrankungen beobachtet worden, wobei die Dermatose hier offensichtlich als direkte Folge der meist fortgeschrittenen Grunderkrankungen entstanden war und nicht als Folge der Behandlung. Entzündliche Darmerkrankungen, z.B. der Morbus Crohn und die Colitis ulcerosa, oder die Tuberkulose mit Befall inguinaler oder axillärer Lymphknoten sind entsprechende Beispiele, die in der Literatur beschrieben worden sind [13-17].

Pathogenetisch zeichnet sich das primäre Lymphangioma circumscriptum durch lymphatische Zisternen aus, die subkutan im Bereich der tiefen Faszien lokalisiert sind. Diese Zisternen gelten als embryonale Entwicklungsanomalien und sind nur mit den Lymphgefäßen der retikulären Dermis verbunden, während der Anschluss an das tiefe lymphatische System fehlt. Bei Kontraktur der Zisternen steigt der Druck im Bereich der retikulären Lymphbahnen. Wird dabei die Elastizität der Gefäße dauerhaft überfordert, entwickeln sich Ektasien [18]. Einer anderen Auffassung zufolge wird das primäre Lymphangioma circumscriptum auf Grund histopathologischer Befunde als autonome Fehlbildung im Sinne einer echten Neoplasie verstanden [2]. Bei der erworbenen Form des Lymphangioma circumscriptum wird die Druckerhöhung im lymphatischen Gefäßsystem durch therapeutische Maßnahmen im Zusammenhang mit Tumorerkrankungen oder durch entzündlich bedingte Schädigungen der Lymphbahnen verursacht. Offensichtlich führt dabei insbesondere die Kombination chirurgischer und radiologischer Therapieverfahren besonders häufig zur Entwicklung der hier unbestritten vorliegenden Lymphangiektasien [3,19].

Bei Kenntnis einer vorausgegangenen onkologischen Therapie und bei Auftreten gruppiert stehender, häufig konfluierender Bläschen in genitaler oder thorakaler Lokalisation dürfte die Diagnose eines erworbenen Lymphangioma circumscriptum im Allgemeinen keine Schwierigkeiten bereiten. Nichtsdestotrotz wurde der dermatologische Befund bei zahlreichen Patienten zunächst als Condylomata acuminata fehlgedeutet [5]. Zu weiteren klinischen Differenzialdiagnosen werden die Mollusca contagiosa, der Morbus Fox-Fordyce und die Hidradenitis suppurativa gezählt [8].

Die Behandlung der erworbenen Form des Lymphangioma circumscriptum ist schwierig, wobei unterschiedliche therapeutische Verfahren angewendet worden sind. Die chirurgische Exzision, soweit sie sinnvoll möglich ist, zeichnet sich durch eine hohe Rezidivrate aus [5]. Die Elektrokoagulation oder die Dermabrasio sind weitere chirurgische Verfahren, die vereinzelt empfohlen worden sind $[15,20]$. Erfolg versprechender als die herkömmlichen chirurgischen Verfahren ist hingegen die Laser-Therapie, die in den letzten Jahren verstärkt auch zur Behandlung des erworbenen Lymphangioma circumscriptum eingesetzt wor- den ist. Neben dem Argon-Laser wurde der Erbium:YAG-Laser und besonders der $\mathrm{CO}_{2}$-Laser verwendet. Die Laser-Therapie ist auch beim Lymphangioma circumscriptum ein den Patienten nur wenig belastendes Behandlungsverfahren, deren Anwendung beliebig wiederholt werden kann und das sich darüber hinaus durch eine nur geringe Rezidivrate der Lymphangiektasien auszeichnet [20-23].

\section{Literatur}

${ }^{1}$ Mentzel T, Kutzner H. Lymphgefäßtumoren der Haut und des Weichgewebes. Pathologe 2002; 23: 118 - 127

2 Eilers J, Daum S, Haas N. Lymphangioma circumscriptum der Zunge. Akt Dermatol 1988; 14: $108-110$

${ }^{3}$ Weyers W, Nilles M, König M. Lymphangioma circumscriptum cysticum nach chirurgischer und radiologischer Therapie. Hautarzt 1990; 41: $102-104$

4 Tulasi NR, John A, Chauhan I et al. Lymphangioma circumscriptum. Int J Gynecol Cancer 2004; 14: 564-566

5 Vlastos, AT , Malpica A, Follen M. Lymphangioma circumscriptum of the vulva: a review of the literatur. Obstet Gynecol 2003; 101: $946-$ 954

${ }^{6}$ Celis AV, Gaughf CN, Sangueza OP et al. Acquired lymphangiectasis. South Med J 1999; 92: 69-72

${ }^{7}$ Fisher I, Orkin M. Acquired lymphangioma. Arch Derm 1970; 101: $230-234$

${ }^{8}$ LaPolla J, Foucar E, Leshin B et al. Vulvar lymphangioma circumscriptum: a rare complication of therapy for squamous cell carcinoma of the cervix. Gynecol Oncol 1985; 22: $363-366$

9 Prioleau PG, Santa CruzDJ. Lymphangioma circumscriptum following radical mastectomy and radiation therapy. Cancer 1978; 42: 19891991

${ }^{10}$ Leshin B, Whitacker DC, Foucar E. Lymphangioma circumscriptum following mastectomy and radiation therapy. J Am Acad Dermatol 1988; 15: $1117-1119$

${ }^{11}$ Gupta S, Radotra BD, Javaheri SM. Lymphangioma circumscriptum of the penis mimicking venereal lesions. JEADV 2003; 17: 598-600

12 Mallett RB, Curley GK, Mortimer PS. Acquired lymphangioma: report of four cases and discussion of pathogenesis. Br J Deramtol 1992; 126: $380-382$

${ }^{13}$ Drago F, Rampini P, Muzio G et al. Lymphangioma circumscriptum of the penis. Acta Derm Venereol (Stockh) 1997; 77: 252

${ }^{14}$ Handfield-Jones SE, Prendiville WJ, Norman S. Vulvar lymphangiektasia. Genitourin Med 1989; 65: 335-337

${ }^{15}$ Heuvel NV, Stolz E, Notowicz A. Lymphangiektases of the vulva in a patient with lymph node tuberculosis. Int J Dermatol 1979; 18: 65-66

${ }^{16} \mathrm{Mu}$ XC, Tran TA, Dupree M et al. Acquired vulvar lymphangioma mimicking genital warts. A case report and review of the literature. J Cutan Pathol 1999; 26: 150-154

17 Sood M, Mandal AK, Ganesh K. Lymphangioma circumscriptum of the vulva. J Indian Med Assoc 1991; 89: 262 - 263

18 Whimster IW. The pathology of lymphangioma circumscriptum. Br J Dermatol 1976; 94: 473-486

${ }^{19}$ Peachy RO, Limm CC, Whimster IW. Lymphangioma of the skin: a review of 65 cases. Br J Dermatol 1970; 83: 519-527

${ }^{20}$ Ochsendorf FR, Kaufmann R, Runne U. Die Therapie superfizieller Lymphangiome: Was ist möglich? Akt Dermatol 2004; 30: 446

${ }^{21}$ Bailin PL, Kantor GR, Wheeland RG. Cabon dioxide laser vaporization of lymphangioma circumscriptum. J Am Acad Dermatol 1986; 14: $257-262$

${ }^{22}$ Landthaler M, Haina D, Waidelich W, Braun-Falco O. Behandlung zirkumskripter Lymphangiome mit dem Argonlaser. Hautarzt 1982; 33 : 266-270

${ }^{23}$ Eliezri Y, Sklar JA. Lymphangioma circumscriptum: review and evaluation of carbon dioxide vaporization. Dermatol Surg Oncol 1988; 14 : 357-364 